\title{
TRATAMENTO CIRÚRGICO DA ATRESIA DE VIAS BILIARES: A EXPERIÊNCIA DO HOSPITAL MUNICIPAL JESUS, 1997-2000
}

\author{
SURGICAL TREATMENT OF BILIARY ATRESIA: \\ THE HOSPITAL MUNICIPAL JESUS EXPERIENCE 1997-2000
}

\author{
Lisieux Eyer de Jesus, TCBC/RJ' \\ Paulo Cesar Costa Monteiro ${ }^{1}$
}

\begin{abstract}
RESUMO: Objetivo: Analisar a indicação, resultados e limitações da portoenterostomia para o tratamento da atresia de vias biliares em hospital infantil terciário no Rio de Janeiro-RJ/Brasil. Método: Foram estudados prospectivamente oito pacientes submetidos à portoenterostomia do tipo Kasai 1, num período de três anos (1997-2000). Todos foram submetidos à antibioticoterapia profilática, uso de colerético, reavaliação médica mensal nos primeiros seis meses de seguimento e reavaliação laboratorial, ultra-sonográfica e através de endoscopia digestiva alta em prazos programados. Resultados: Em cinco pacientes foi possível obter drenagem biliar estável e satisfatória por > 6 meses pós-operatórios. Quatro faleceram no período de observação (um por insuficiência hepática, um por colangite, dois por hemorragias digestivas, inclusive os três pacientes em que não foi obtida drenagem biliar satisfatória). Quatro persistem em bom estado geral, anictéricos, um deles com fibrose hepática residual grave e provável indicação futura de transplante hepático. Detectamos problemas graves com relação ao encaminhamento tardio de doentes para tratamento e para disponibilizar transplante hepático quando necessário. Conclusões: Os resultados da portoenterostomia são compensadores quando é possível obter bom débito biliar no pós-operatório. Em pacientes em que não é obtida boa drenagem biliar o pós-operatório é tormentoso e o óbito é esperado até o segundo ano de vida sem o uso de transplante hepático. É necessário disponibilizar transplante hepático pediátrico em nosso meio e conscientizar a comunidade médica para o encaminhamento precoce de bebês ictéricos além dos primeiros 15 dias de vida para investigação e tratamento em unidades especializadas.
\end{abstract}

Descritores: Atresia de vias biliares; Portoenterostomia; Icterícia neonatal; Operação de Kasai.

\section{INTRODUÇÃO}

A cirurgia das vias biliares na criança tem um impacto consideravelmente diferente daquele encontrado nos adultos. Este fato está relacionado com a incidência proporcionalmente menor de doenças biliares nesta faixa etária. Além disto, existem implicações muito diferentes no que se refere à manipulação das estruturas anatômicas envolvidas, extremamente delicadas. Simultaneamente há dificuldades de treinamento pela menor exposição dos especialistas a biliopatias, implicações em prazo muito prolongado dos resultados obtidos e envolvimento preferencial das estruturas do pedículo hepático na maioria dos casos, excluindo casos de colelitíase não complicada. A atresia de vias biliares é a doença biliar mais comum em cirurgia pediátrica $(89 \%$ dos casos de doença biliar cirúrgica em crianças $)^{1}$. Calcula-se que sua incidência populacional no ocidente seja em torno de 1:15.000 nascidos vi$\operatorname{vos}^{1}$. As dificuldades técnicas para o tratamento são significativas, e ainda há controvérsias a respeito. Há implicações da faixa etária dos pacientes operados com relação aos resultados obtidos e complicações graves no seguimento pós-operatório, notadamente colangite recorrente.

Abordaremos aqui a experiência do Hospital Municipal Jesus nos útimos três anos no tratamento cirúrgico da atresia de vias biliares. Consideramos esta comunicação importante em face de não termos encontrado casuísticas nacionais recentes disponíveis em literatura. Desta forma, pretendemos discutir aqui os problemas apresentados com relação ao encaminhamento tardio de pacientes, as dificul-

1. Cirurgiãos Pediátricos do Hospital Municipal Jesus e Hospital Universitário Antônio Pedro da UFFRJ.

Recebido em 25/7/2000

Aceito para publicação em 14/11/2000

Trabalho realizado no Serviço de Cirurgia Pediátrica do Hospital Municipal Jesus, Rio de Janeiro. 
dades com relação a complicações pós-operatórias e o encaminhamento final de pacientes que necessitem de transplante hepático.

\section{MÉTODO}

No período de junho 1997 a junho 2000 foram operados no Hospital Municipal Jesus oito casos de atresia de vias biliares (AVB), todos com portoenterostomia do tipo Kasai I pelo mesmo cirurgião (LEJ), à exceção do paciente $\mathrm{n}$ o 5. Destacamos que neste mesmo período foram recebidos no hospital quatro outros portadores da doença (incidência global da doença na instituição de quatro casos/ ano) acima da idade em que padronizamos o limite para executar portoenterostomia (quatro meses de idade). Estes últimos não foram operados e receberam acompanhamento clínico de suporte, aguardando oportunidade futura de transplante hepático que, infelizmente, não foi obtido para nenhuma das crianças.

Estudamos prospectivamente a evolução dos oito pacientes submetidos à cirurgia de Kasai (portoenterostomia) neste período, com seguimento pós-operatório variando entre três e 31 meses. Todos foram submetidos à portoenterostomia do tipo Kasai I, drenagem do espaço sub-hepático com dreno de Penrose e biópsia de fígado. Utilizou-se rotineiramente antibiotico parenteral inicial por três semanas pós-operatórias (PO) com ceftriaxona, $100 \mathrm{mg} / \mathrm{kg} / \mathrm{d}$. Esta conduta foi alterada nos casos de infecção instalada em vigência do tratamento. A profilaxia antiinfecciosa foi continuada com cefalexina oral em dose única noturna $(25 \mathrm{mg} / \mathrm{kg})$ por seis meses, associada ao ácido ursodeoxicólico $(15 \mathrm{mg} / \mathrm{kg} / \mathrm{d})$ e complexo vitamínico ADEK. Todos os pacientes operados foram submetidos a acompanhamento ambulatorial mensal durante o primeiro semestre pós-operatório e trimestral a seguir. Os responsáveis receberam reiteradamente instruções para retorno hospitalar emergencial no caso de observarem sintomas sugestivos de colangite. Os exames complementares utilizados no pós-operatório consistiram em ultra-sonografias (US) seriadas para verificar evolução do aspecto do fígado e sinais de hipertensão portal, exames laboratoriais para vigilância de função hepática e endoscopia digestiva alta após o $1^{\circ}$ ano de vida, para pesquisar sinais de hipertensão portal. Os familiares dos doentes foram informados, ainda no préoperatório, quanto à gravidade do problema e à necessidade eventual de transplante hepático futuro.

\section{RESULTADOS}

Observamos no grupo estudado que houve encaminhamento tardio ao serviço (Tabela 1). A maioria foi avaliada por pediatras generalistas em curso de puericultura. Dois pacientes foram inicialmente levados para o departamento de doenças infectoparasitárias (DIP) para investigação de infecção congênita como causa de icterícia perinatal persistente. Apenas um paciente (caso 1) foi conduzido imediatamente para parecer cirúrgico buscando exclusão de atresia de vias biliares. Após admissão em nosso serviço, os doentes foram rapidamente submetidos a exames para avaliação cirúrgica, e logo operados (tempo decorrido entre exames e operação dois a 20 dias, média 7,3 dias.

Todas as crianças operadas apresentavam estado geral e nutricional satisfatórios, icterícia de leve à moderada (nível pré-operatório médio de bilirrubina total médio 9,9mg.dL, 3,6mg/dL-17,4mg/dL), iniciada no primeiro mês de vida (em média $2^{\text {a }}$ semana), colúria e acolia/hipocolia fecal, níveis altos de fosfatase alcalina sérica (mínimo 342UI/dL, máximo 916UI/dL) e pequena elevação dos níveis séricos de transaminases: AST (mínimo 102UI/dL, máximo 354UI/dL) e ALT (mínimo 66UI/dL, máximo 199UI/dL). Hepatomegalia com fígado endurecido foi uma constante e quatro crianças (casos 2/3/7/8) apresentavam também exame físico sugestivo de esplenomegalia. Realizou-se ultra-sonografia em todas as crianças, não pesquisamos a presença do triângulo hiperecogênico hilar ${ }^{8}$ (Tabela 2). $\mathrm{O}$ grupo foi submetido à cintilografia de vias biliares, e o resultado mostrou ausência de eliminação de contraste para as alças intestinais após 24h (casos 1/3/5/6/ 7/8), 4h (caso 4) e 6h (caso 2) de exame, sem preparo prévio com barbitúricos. Não utilizamos tubagem duodenal, biópsia hepática pré-operatória, ressonância magnética ou colangiografia endoscópica. Pesquisou-se em todos os pacientes a presença de infecções congênitas (testes TOR$\mathrm{CH}$, pesquisa de hepatites A,B e C, pesquisa de anticorpos anti-HIV e VDRL), com resultados negativos.

Os achados operatórios estão relacionados na Tabela 3. Foi utilizada corticoterapia (pulsoterapia com prednisolona $)^{2}$ no pós-operatório imediato em todos os casos, exceto nos pacientes $n^{\text {os }} 3$ e 6 , que apresentaram drenagem biliar espontânea e estável sem qualquer problema detectável desde o 3o dia pós-operatório (PO). Todos apresentaram drenagem biliar presente precocemente (em média desde o 3ำ dia PO), perceptível através de evacuação de fezes coradas, mas o débito biliar foi instável, alternando fezes normocoradas e hipocólicas nos pacientes 1/4/6/8.

Os resultados de seguimento são mostrados nas Tabelas 4, 5, 6 e 7. Encontrou-se leucocitose intensa em sete dos oito pacientes no pós-operatório imediato, não correlacionável a episódios infecciosos detectáveis ou a outros

\section{Tabela 1}

Idade de apresentação dos pacientes portadores de atresia de vias biliares (AVB) para tratamento

\begin{tabular}{c|lccc}
\hline Paciente & $\begin{array}{c}\text { Idade avaliação } \\
\text { médica inicial }\end{array}$ & $\begin{array}{c}\text { Idade referência } \\
\text { ao cirurgião }\end{array}$ & $\begin{array}{c}\text { Idade } \\
\text { operação }\end{array}$ \\
\hline 1 & $53 \mathrm{~d}$ (dip)* & $53 \mathrm{~d}$ & $73 \mathrm{~d}$ \\
2 & $79 \mathrm{~d}$ (dip) & $117 \mathrm{~d}$ & $119 \mathrm{~d}$ \\
3 & $45 \mathrm{~d}$ (ped geral) & $133 \mathrm{~d}$ & $138 \mathrm{~d}$ \\
4 & 20d (ped geral) & $61 \mathrm{~d}$ & $72 \mathrm{~d}$ \\
5 & $49 \mathrm{~d}$ (gastroped) & $57 \mathrm{~d}$ & $64 \mathrm{~d}$ \\
6 & 20d (ped geral) & $47 \mathrm{~d}$ & $51 \mathrm{~d}$ \\
7 & $51 \mathrm{~d}$ (ped geral) & $55 \mathrm{~d}$ & $70 \mathrm{~d}$ \\
8 & 13d (ped geral) & $94 \mathrm{~d}$ & $99 \mathrm{~d}$ \\
\hline
\end{tabular}

* dip = doenças infectoparasitárias. 
fatores conhecidos e sem quaisquer manifestações clínicas anormais. Rotineiramente persistem níveis aumentados de fosfatase alcalina sérica., independentemente do sucesso ou não da drenagem cirúrgica. A resposta tardia foi insa-

\section{Tabela 2}

Resultados de exames ultra-sonográficos.

Em 2 pacientes foram realizadas duas ultra-sonografias (casos 6 e 7) e houve discordância de laudos.

Optamos por assinalar aqui os resultados do último exame realizado anteriormente à operação

\begin{tabular}{lc}
\hline Achados US & No pacientes \\
\hline Hepatomegalia & 8 \\
Esplenomegalia (VR 40-65 mm) & 2 \\
Ausência de dilatação de vias biliares & 3 \\
Vias biliares não visualizadas & 3 \\
Cisto na topografia de colédoco & 1 (caso 7) \\
Vesícula biliar presente & 1 \\
Vesícula biliar diminuída & 1 \\
Vesícula biliar não visualizada & 6 \\
Suspeita de atresia de vias biliares & 2 (caso 6/8) \\
Hipertensão portal & 2 (caso 2/8) \\
\hline
\end{tabular}

\section{Tabela 3}

Achados cirúrgicos

\begin{tabular}{l|c}
\hline Achados cirúrgicos & No pacientes \\
\hline $\begin{array}{l}\text { Atresia VBEH subtipo IIIc da classificação } \\
\text { japonesa3 (formas “não corrigíveis") }\end{array}$ & 8 \\
Vesícula fibrosada e sem luz/ rudimentar & 7 \\
Anomalia da a hepática & 2 \\
Adenomegalia extensa pedículo biliar & 6 \\
Cisto biliar conectado ao ducto cístico & 1 (caso 7) \\
Vesícula biliar patente, hidrópica & 1 (caso 7) \\
Hipertensão portal estabelecida & 1 (caso 8) \\
\hline
\end{tabular}

$V B E H=$ vias biliares extra-hepáticas. tisfatória em quatro pacientes (dois óbitos por complicações de cirrose biliar em pacientes com drenagem instável no PO (casos 1/6), um óbito por colangite em PO precoce (caso 2) e um paciente com resultados ruins da drenagem, que evoluiu com colangite recorrente e óbito por insuficiência hepática (caso 8). Possivelmente o atendimento em tempo hábil no caso no 2 seria capaz de evitar o êxito letal, em paciente com boa evolução prévia e morte por sepse após episódio de colangite com tratamento tardio (72h após o início de episódio febril). Três pacientes tiveram resultado ótimo, evoluindo sem problemas clínicos relevantes (sete meses a dois anos PO). Um paciente teve resultado do tratamento cirúrgico considerado razoável, com evolução clínica satisfatória, mas provável indicação tardia de transplante por fibrose hepática residual. Destacamos aqui as dificuldades verificadas para os dois doentes que tiveram indicação firmada de transplante hepático com menos de um ano de idade, falecendo enquanto aguardavam tratamento definitivo em serviço de referência nacional. Ambos foram a óbito por hemorragia digestiva devido a varizes de esôfago sangrantes. Um destes (caso 1) faleceu com estado geral satisfatório e agendamento para transplante inter vivos para prazo de um mês após a data do óbito e outro (caso 6) faleceu ainda sem agendamento para transplante, apresentando problemas clínicos graves por desnutrição (peso em torno de $7 \mathrm{~kg}$ aos 13 meses de idade) e colangites recorrentes. Nos demais pacientes não há evidências endoscópicas (casos 3 e 4) ou ultra-sonográficas de hipertensão portal. Os pacientes 5 e 7 aguardam ainda avaliação endoscópica.

\section{DISCUSSÃO}

Embora a casuística apresentada seja pequena em termos numéricos, é representativa pela relativa raridade da doença em nosso meio. Em inquérito nacional realizado recentemente ${ }^{4}$ foi sugerida a incidência média de 3,5 casos cirúrgicos/ano nos serviços de cirurgia pediátrica brasileiros envolvidos no tratamento da atresia de vias biliares, semelhante à nossa casuística.

Modernamente alguns autores sugerem que o prognóstico tardio para os portadores de atresia de vias biliares tratados pela portoenterostomia, determinado pela capa-

Tabela 4

Resultados pós-operatórios imediatos

\begin{tabular}{|c|c|c|}
\hline Paciente & Resultado imediato & Uso corticóide \\
\hline 1 & Drenagem biliar a partir do $3^{\circ} \mathrm{DPO}$, instável, alternando fezes coradas/ hipocólicas & Pulsoterapia \\
\hline 2 & Drenagem biliar efetiva mantida desde $4^{\circ} \mathrm{PO}$ & Pulsoterapia \\
\hline 3 & Drenagem biliar efetiva mantida desde $2^{\circ} \mathrm{DPO}$ & Não utilizado \\
\hline 4 & Drenagem biliar a partir do $2^{\circ} \mathrm{DPO}$, inicialmente instável, estabilizada 3 meses PO & Pulsoterapia \\
\hline 5 & Drenagem biliar efetiva a partir do $2^{\circ} \mathrm{DPO}$ & Não utilizado \\
\hline 6 & Drenagem biliar a partir do $3^{\circ} \mathrm{DPO}$, instável & Pulsoterapia \\
\hline 7 & Drenagem biliar estável a partir do $2^{\circ} \mathrm{DPO}$ & Pulsoterapia \\
\hline 8 & Drenagem biliar instável a partir $3^{\circ} \mathrm{DPO}$ & Pulsoterapia \\
\hline
\end{tabular}


Tabela 5

Níveis de bilirrubina total no pós-operatório (mg/dL)

\begin{tabular}{c|l|l|c|c}
\hline Paciente & Resultado final & Bb total 1m PO & Bb totaal $3 m P O$ & $B b$ total $6 m$ PO \\
\hline 1 & Óbito $13 \mathrm{~m}$ PO & 3,5 & 11,5 & 7,7 \\
2 & Óbito $2 \mathrm{~m}$ PO & Óbito 2 ${ }^{\circ}$ mês pós-operatório, BbT 2,4mg/dL & 1,1 \\
3 & Ótimo, seguimento $26 \mathrm{~m}$ & Anictérico & 1,3 & 2,1 \\
4 & Bom, seguimento $20 \mathrm{~m}$ & 6,7 & 4,0 & Anictérico \\
5 & Ótimo, seguimento $18 \mathrm{~m}$ & 3,1 & 3,9 & 6,2 \\
6 & Óbito $11 \mathrm{~m}$ PO & 8,2 & 1,1 & 0,8 \\
7 & Ótimo, seguimento $7 \mathrm{~m}$ & 5,1 & Óbito 2 ${ }^{\circ}$ mês pós-operatório, Bb $>30 \mathrm{mg} / \mathrm{dL}$ &
\end{tabular}

Tabela 6

Complicações pós-operatórias

\begin{tabular}{|c|c|c|c|}
\hline Paciente & Colangite & Hipertensão portal & Outros \\
\hline 1 & $\begin{array}{l}2 \text { episódios, em vigência de } \\
\text { antibioticoprofilaxia }\end{array}$ & $\begin{array}{l}1 \text { a } \mathrm{PO}, \text { hemorragia digestiva por } \\
\text { varizes de esôfago e óbito }\end{array}$ & - \\
\hline 2 & $\begin{array}{l}2 \text { episódios, } 10 \mathrm{~d} \text { PO e } 40 \mathrm{~d} \mathrm{PO}, \\
\text { óbito no } 2^{\circ} \text { episódio }\end{array}$ & - & - \\
\hline 3 & Sem complicações, 26 m PO & & \\
\hline 4 & - & $\begin{array}{l}\text { Esplenomegalia assintomática, } \\
\text { veia porta } 5 \mathrm{~mm}\end{array}$ & Fibrose hepática residual grave \\
\hline 5 & Sem complicações, 18 m PO & & \\
\hline 6 & $\begin{array}{l}4 \text { episódios }>5 \mathrm{~m} \text { PO, } 1 \text { episódio } \\
\text { de colangite fúngica }\end{array}$ & $\begin{array}{l}\text { Esplenomegalia > 6m PO. } \\
\quad \text { Hemorragia digestiva } 11 \mathrm{~m} \mathrm{PO} \mathrm{e}\end{array}$ & $\begin{array}{l}\text { Déficit crescimento severo, ascite, } \\
\text { cirrose hepática sintomática. }\end{array}$ \\
\hline 7 & Sem complicações, PO & & \\
\hline 8 & 2 episódios, 3 e 6 semanas PO & - & $\begin{array}{l}\text { Hemorragia digestiva PO imediato, } \\
\text { gastropatia de stress. Óbito } 2 \mathrm{~m} \text { PO, } \\
\text { insuficiência hepática. }\end{array}$ \\
\hline
\end{tabular}

cidade de obter drenagem biliar efetiva e estável, pode ser previsto já a partir do seguimento inicial (seis meses) por exames simples. A persistência de níveis baixos $(<2,5 \mathrm{mg} / \mathrm{dL})$ de bilirrubina total, ausência de colangites e índices pouco elevados de transaminases podem definir um paciente específico como de provável evolução satisfatória a longo prazo $^{6,10}$.

A idade de encaminhamento das crianças com atresia de vias biliares para tratamento cirúrgico é considerada crucial na determinação dos resultados da operação do tipo portoenterostomia, ainda que individualmente possam ser obtidos bons resultados em pacientes mais velhos. Embora variáveis, as respostas da drenagem são melhores para os casos operados com menos de 60 dias de idade com relação às crianças cuja intervenção foi efetuada após este prazo (sobrevida a longo prazo variando de $46 \%$ a $73 \%$ dos pacientes operados precocemente, e em torno de $1 / 3$ dos pacientes operados após 60 dias de vida $)^{5-7,10}$. Pudemos registrar o direcionamento tardio dos pacientes para operação, com la- tência média entre a primeira avaliação pediátrica da criança ictérica e o encaminhamento ao cirurgião de 36 dias. Os problemas não são atribuíveis à busca tardia de atenção médica pelos pais (média de idade da criança na primeira avaliação médica quanto à icterícia 41 dias) ou ao atraso de realização da operação (prazo médio da intervenção cirúrgica após o encaminhamento do paciente ao cirurgião é de 7,2 dias). É possível deduzir que há a necessidade de conscientização maior do problema pelos pediatras e/ou a necessidade de uma reorganização dos serviços de saúde para a avaliação e seguimento efetivo de neonatos ictéricos no primeiro mês de vida, já que foi o tempo de latência entre a avaliação médica inicial e o encaminhamento para tratamento cirúrgico o responsável pelo retardo terapêutico. O encaminhamento tardio é problema em todo o território nacional, uma vez que a média de atendimento dos pacientes pelos serviços cirúrgicos é de 10 semanas de vida e média etária no ato operatório de 12,6 semanas ${ }^{4}$. A divulgação quanto à necessidade de exclusão precoce de atresia de vias biliares, em 
Tabela 7

Resultados finais

\begin{tabular}{c|c}
\hline Paciente & \multicolumn{1}{c}{ Resultado } \\
\hline 1 & Óbito 13 meses pós-operatórios, hemorragia digestiva (varizes de esôfago), transplante hepático agendado. \\
2 & Óbito $2^{\circ}$ mês pós-operatório, episódio de colangite tratado após $48 \mathrm{~h}$ de evolução sem atendimento médico, \\
choque séptico na admissão. & Seguimento 2 anos, assintomático, ótimo estado geral, crescimento normal. \\
3 & Seguimento 20 meses, bom estado geral, icterícia leve, hepatomegalia, crescimento satisfatório. Provável \\
4 & necessidade futura de transplante hepático, em follow-up de vigilância em serviço especializado. \\
5 & Seguimento 18 meses, ótimo estado geral, assintomático, crescimento normal. \\
6 & Óbito 13 meses idade, hemorragia digestiva por varizes de esôfago, aguardando transplante hepático. Mau \\
7 & estado geral e nutricional, vários episódios de colangite a partir de 5 m PO. \\
8 & Seguimento 7 meses PO, ótimo estado geral, assintomático, crescimento normal. \\
& Óbito 2 meses PO. Mau estado geral, cirrose biliar estabelecida, icterícia persistente, 2 episódios de colangite. \\
Causa mortis insuficiência hepática.
\end{tabular}

qualquer neonato com icterícia não hemolítica persistente após os 15 dias de vida, seria eficaz para reduzir o problema, selecionando ainda no primeiro mês estes pacientes para referência de urgência a unidades especializadas.

As crianças portadoras de atresia de vias biliares, ao contrário daquelas vítimas de hepatites por infecções congênitas e distúrbios metabólicos em geral se apresentam em bom estado geral e nutricional, e com níveis pouco elevados de transaminases, atípicos das hepatites neonatais. As taxas de bilirrubina não são, ao contrário do que se possa julgar desavisadamente, excessivamente elevadas, e é típico um aumento da fosfatase alcalina e outros marcadores de lesões biliares obstrutivas. Notadamente, sinais clínicos (hipocolia fecal e colúria) e laboratoriais (elevação de fosfatase alcalina e gamaglutamil transferase) de colestase não conferem certeza de condições obstrutivas mecânicas das vias biliares extra-hepáticas, e podem ser encontrados em hepatites em geral, como sinalizantes de colestases intra-hepáticas. Esplenomegalia é apresentada por vários autores como um achado sugestivo de infecção perinatal, mas em nossa casuística não se mostrou uma boa referência diferencial, sendo sugerida em metade dos casos de atresia de vias biliares, em dois deles confirmada por métodos de imagem. É muito importante considerar que os exames marcadores de infecção perinatal (testes Torch e outros) não devem retardar a operação em casos fortemente sugestivos de AVB.

Consideramos a ultra-sonografia importante como exame de triagem para detectar outras doenças biliares possíveis nesta faixa etária (perfuração espontânea de vias biliares e cisto de colédoco, especificamente). No entanto, o ultra-som não ajuda definitivamente no diagnóstico quanto à presença ou à ausência de atresia de vias biliares, e foi responsável pelo atraso no diagnóstico em vários casos, quando evidenciou a vesícula biliar e emitiu laudos de "ausência de dilatação de vias biliares", induzindo à falsa impressão de normalidade, ou sugeriu "cisto de colédoco" (na verdade lago biliar, caso 7). Modernamente vem sendo investigada a presença de área triangular ecogênica de fi- brose hilar como sinal precoce da doença ${ }^{8}$, detectável em exame de ultra-sonografia, mas este dado ainda se encontra insuficientemente estudado e pouco popularizado entre os radiologistas.

A cintigrafia de vias biliares tem se mostrado eficaz para o diagnóstico indireto da doença, pela ausência do traçador no trato digestivo após $24 \mathrm{~h}$ da injeção da substância. O maior problema encontrado em nossos casos foi a execução inadequada do exame em duas oportunidades, com a leitura excessivamente prematura dos resultados com 4h e 6 h de exposição, tempo insuficiente para o diagnóstico diferencial com colestases acentuadas. Não utilizamos tubagem duodenal, pois duvidamos da confiabilidade dos resultados em nossas circunstâncias operacionais, seja por dificuldade em manter a localização do cateter nasoduodenal estável a longo prazo (12h), seja para providenciar a logística de pessoal e laboratório para o recolhimento periódico regular correto das amostras ${ }^{9}$. Não fazemos biópsia hepática pré-operatória ou ressonância magnética. Acentuamos aqui que, corroborando os ensinamentos já clássicos para a abordagem da atresia de vias biliares, a exploração operatória precoce está indicada em todos os casos em que haja uma suspeita bem fundamentada, a fim de não abrir mão da operação numa faixa etária ideal e de maior eficácia para o tratamento.

Todos os pacientes eram portadores de atresia completa das vias biliares até o nível do "porta hepatis", com atresia da vesícula biliar (exceto paciente 7). Foi muito comum a presença de alterações inflamatórias e adenopatias no pedículo hepático. A incidência de anomalias da artéria hepática foi maior que a encontrada na população geral (2/8 pacientes), mas não encontramos nenhum caso anomalia de veia porta. O uso de prednisolona como colerético foi efetivo no período pós-operatório imediato, sob a forma de pulsoterapia, em média a partir do 5o dia após o ato cirúrgico ${ }^{2}$.

Foi possível obter drenagem biliar precoce em todos os casos, mas esta drenagem não se manteve estável após 15 dias em três dos oito pacientes, todos os quais persistiram clinicamente ictéricos e apresentaram episódios de 
colangite grave na evolução, fortemente relacionados com um mau prognóstico, fator em que nossa avaliação coincide com a de diversos autores ${ }^{3-7 ; 10}$. Colangite foi verificada apenas em um dos cinco pacientes em que a drenagem biliar inicial foi satisfatória e estável. Entre eles, houve apenas um óbito, potencialmente evitável (colangite não tratada em tempo hábil), três crianças se encontram em excelente estado geral, com função hepática estável e de boa qualidade de vida (seguimento de sete a dois meses PO) e um está estável, com qualidade de vida satisfatória, embora com fibrose hepática residual extensa e previsão quanto à necessidade futura de transplante, em período de tempo indeterminado (seguimento de 20 meses PO).

Com relação às drenagens cirúrgicas bem-sucedidas verificamos respostas compensadoras das portoenteroanastomoses, capazes de proporcionar boa ou razoável qualidade de vida. A ausência de indicação de transplante hepático precoce é também uma vantagem, uma vez que é reconhecido o prognóstico pior do transplante em crianças menores de um ano de idade. Naqueles em que não foi possível obter bons resultados com o tratamento cirúrgico, o seguimento PO foi tormentoso, em especial pela sobrevinda de colangites recorrentes e suas complicações. Não temos dados para estabelecer uma comparação entre estes pacientes e o padrão clínico evolutivo da história natural da doença em nosso meio, que consideraríamos interessante para fundamentar uma discussão quanto à validade da portoenteroanastomose, comparando-a à não execução de qualquer outra operação para a realização de um transplante hepático futuro.

Paradoxalmente, a faixa etária média no momento do tratamento cirúrgico nos casos de drenagens ineficazes versus eficazes em nosso serviço foi de 74 e 93 dias de vida, respectivamente. Esta incapacidade de correlacionar idade na época do ato cirurgico e resultados em nossa casuística possivelmente tem relação com a pequena amostragem, mas este mesmo aspecto aparece no Inquérito Nacional já cita$\mathrm{do}^{4}$, e aí talvez possa ser atribuído à tendência ao atendimento mais tardio dos doentes em nosso meio, como já discutimos anteriormente. Todos os pacientes foram operados segundo o mesmo padrão técnico e $88 \%$ pelo mesmo cirur- gião, com igual protocolo para seguimento e tratamento. $\mathrm{O}$ fator colangite foi representativo de mau prognóstico nos casos de drenagem ineficaz, mas somos incapazes de definir se a presença de colangite precoce determinou a drenagem biliar ineficaz destes doentes ou se, inversamente, a drenagem ineficiente proporcionou o maior risco de colangite. No entanto, a boa qualidade de vida nos casos bem-sucedidos nos sugere que a indicação cirúrgica da portoenteroanastomose não deve ser restrita aos dois primeiros meses de vida, embora restrinjamos, em nosso serviço, as indicações aos pacientes até quatro meses de idade.

Finalmente, destacamos as dificuldades enfrentadas em nosso meio para atender aos pacientes pediátricos com indicação de transplante hepático, em especial em lactentes menores de um ano ou crianças abaixo de $10 \mathrm{~kg}$ de peso, circunstâncias em que as dificuldades técnicas são maiores, mesmo em centros mundiais de excelência em transplante pediátrico. Destacamos o fato de que nenhum dos pacientes portadores de atresia de vias biliares do Hospital Municipal Jesus com indicação de transplante hepático, entre pacientes pós-operados e os não tratados para a doença, logrou ser transplantado em tempo hábil. Sete pacientes (casos 1, 6 e 8 e todos os quatro pacientes não operados) faleceram antes da disponibilidade de transplante.

São necessárias medidas educativas para melhorar o encaminhamento precoce para tratamento definitivo em crianças com atresia de vias biliares. A portoenteroanastomose foi eficaz em $63 \%$ de nossos pacientes para assegurar drenagem biliar e $50 \%$ das crianças operadas sobrevivem com boa qualidade de vida e padrão normal de crescimento e desenvolvimento. A incapacidade de obter drenagem biliar eficaz e estável e a presença de colangites foram fatores negativos cruciais para determinar a sobrevida dos pacientes operados, e a profilaxia com cefalexina oral não foi uniformemente eficiente para diminuir sua incidência. A qualidade de vida dos pacientes com resultados ruins da drenagem é má, e a disponibilidade de transplante hepático pediátrico é francamente insuficiente para suprir a demanda em nosso meio. Sangramento digestivo secundário à hipertensão portal foi fator para $50 \%$ dos óbitos nesta casuística.

\begin{abstract}
Background: Indications, results and limitations of portoenterostomy to treat biliary atresia in a referral pediatric hospital in Rio de Janeiro, Brazil, are discussed. Methods: Eight patients were prospectively evaluated after being submitted to portoenterostomy (Kasai I) in a 3 years period (1997-2000). Seven of then operated by the same surgeon. All patients reserved to prophylactic antibiotics, oral choleretics and underwent a monthly followup for the first six months, and all of them were periodically submitted to laboratory, ultra-sonographic and endoscopid evaluations, following a pre-established schedule. Results: We could obtain a stable biliary drainage for more than 6 post-operative months in 5 patients. There were 4 post-operative deaths ( 1 hepatic insuficiency, 1 cholangitis and 2 esophageal varices ruptures). All three patients with an insuficient biliary drainage died. Four of the five patients with a good and stable biliary drainage are anicteric and in good shape, one of them shows a serious residual hepatic fibrosis. We detected a generally late referral of patients and we could not obtain hepatic transplantation as necessary. Conclusion: Portoenterostomy is a satisfactory operation if we can obtain a satisfactory and stable post-operative biliary drainage. In cases without satisfactory biliary excretion quality of life is unsatisfactory and death occurs in the 2nd year, without hepatic transplantation. It is urgent to offer pediatric hepatic transplantation in a higher scale in Brazil and to educate doctors to send urgently icteric babies more than 15 days old to specialized units to be evaluated and treated precociously enough.
\end{abstract}

Key Words: Biliary atresia/ Portoenterostomy/ Neonatal icterus/ Kasai surgery 


\section{REFERÊNCIAS}

1. Lilly JR, Karrer FM, Hall R et al. The surgery of biliary atresia. Ann Surg, 1989; 210(3):289.

2. Muraji T, Higashimoto Y. The improved outlook for biliary atresia with corticosteroid therapy. J Pediatr Sug 1997; 32(7):1103.

3. Hays DM, Kimura K. Biliary atresia: new concepts of management. Curr Probl Surg 1981; 542.

4. Maksoud JG. Resultados do inquérito Nacional de atresia de vias biliares, apresentado no XX Congresso Brasileiro de Cirurgia Pediátrica, Belo Horizonte, MG, outubro de 1999.

5. Karrer FM, Lilly JR, Stewart BA et al. Biliary atresia registry, 1976 to 1989. J Pediatr Surg 1990; 25(10):1076.

6. Ohi R, Nio M, Chiba T et al. Long-term follow up after surgery for patients with biliary atresia. J Pediatr Surg 1990; 25(4):442.

7. Lugo-Vicente H. Biliary atresia, an overview. http:// home.coqui.net/ titolugo/articles.htm, acessado abril 2000.

8. Park WP, Lee HJ. The ultrasonographic "triangular cord"coupled with gallbladder images in the diagnostic prediction of biliary atresia from infantile intrahepatic cholestasis. J Pedistr Surg 34(11): 1999; 1706.
9. Greene HL, Hellinek GL, Moran R et al. A diagnostic approach to prolonged obstructive jaundice by 24-hour collection of duodenal fluid. J Pediatr 1979; 95(3):412.

10. Subramaniam R, Doig CM, Bowen J et al. Initial response to portoenterostomy determines long-term outcome in patients with biliary atresia. J Pediatr Surg 2000; 35(4):59.

Endereço para correspondência:

Dra. Lisieux Eyer de Jesus

Rua Presidente Domiciano, 52/801

24210-270 — Niterói-RJ

E-mail: lisieux@openlink.com.br 